\title{
CORRECTIONS
}

\section{Fusion for lumbar spinal stenosis?}

Editing of the final sentence in the fourth paragraph of this Editorial by Ashley A Cole (BMJ 2016;353:i3145, doi:10.1136/ bmj.i3145) resulted in it saying the opposite of what was intended. It should read: "but future surgery for degenerative lumbar spinal stenosis without spondylolisthesis should not include fusion." 